\title{
A New Estimation of the Growth Bound of a Periodic Evolution Family on Banach Spaces
}

\author{
Constantin Buse, ${ }^{1}$ Aftab Khan, ${ }^{2}$ Gul Rahmat, ${ }^{2}$ and Afshan Tabassum ${ }^{2}$ \\ ${ }^{1}$ Department of Mathematics, West University of Timisoara, Boulevard V. Parvan No. 4, 300223 Timisoara, Romania \\ ${ }^{2}$ Abdus Salam School of Mathematical Sciences (ASSMS), Government College University, 68-B, New Muslim Town, \\ Lahore 54600, Pakistan
}

Correspondence should be addressed to Constantin Buse; buse1960@gmail.com

Received 20 May 2013; Accepted 26 August 2013

Academic Editor: John R. Akeroyd

Copyright (C) 2013 Constantin Buse et al. This is an open access article distributed under the Creative Commons Attribution License, which permits unrestricted use, distribution, and reproduction in any medium, provided the original work is properly cited.

Let $\mathcal{U}=\{U(t, s)\}_{t \geq s \geq 0}$ be a strongly continuous and $q$-periodic evolution family acting on a complex Banach space $X$. We prove that if $0<p<\infty, x \in X$, and $\sup _{s \geq 0} \sup _{\|x\| \leq 1} \int_{0}^{\infty}\|U(t+s, s) x\|^{p} d t:=C_{p}<\infty$, then the growth bound of the family $\mathcal{U}$ is less than or equal to $-1 /\left(p C_{p}\right)$.

\section{Introduction}

Knowing the rate of convergence of an iterative process, we can control the speed of its convergence. This helps us to obtain the limit of the process, with the desired accuracy, in a determined time. On the other hand, in the study of certain dynamical systems or differential equations, we meet many times $X$-valued functions $f$, defined on $\mathbb{R}_{+}:=[0, \infty)$, having the property that the map $t \mapsto e^{-\omega t}\|f(t)\|$ is bounded on $\mathbb{R}_{+}$, for a suitable real number $\omega$. Here, and in the following, $X$ stands for a real or complex Banach space. Clearly, every $\omega^{\prime} \geq \omega$ has the same property. The infimum of all real numbers $\omega$ which verify the given boundedness condition is called exponential growth of the function $f$. In this note, we obtain an estimate of the exponential growth of a periodic evolution family that satisfies an integral condition, originally given by Datko. The theoretical result allows us to estimate the $L^{2}$-norm of the solution of a periodic Cauchy problem with time-varying coefficients, which is naturally led by the onedimensional heat equation.

The classical theorem of Datko [1] states that a strongly continuous evolution family $\mathcal{U}=\{U(t, s)\}_{t \geq s>0}$, acting on a real or complex Banach space $X$, is uniformly exponentially stable (i.e., there are two positive constants $N$ and $\nu$ ) such that

$$
\|U(t, s)\| \leq N e^{-\gamma(t-s)}, \quad \forall t \geq s \geq 0
$$

if, for some (and then for all) $1 \leq p<\infty$, one has

$$
\sup _{s \geq 0} \int_{0}^{\infty}\|U(t+s, s) x\|^{p} d t<\infty, \quad \forall x \in X .
$$

As usual, the norm of $X$ and of $\mathscr{L}(X)$ is denoted by $\|\cdot\|$. Here, $\mathscr{L}(X)$ denotes the Banach algebra of all the bounded linear operators acting on $X$. By $\sigma(T)$, we denote the spectrum of the linear operator $T$, and when it is bounded, its spectral radius is defined by

$$
r(T):=\sup \{|z|: z \in \sigma(T)\}=\lim _{n \rightarrow \infty}\left\|T^{n}\right\|^{1 / n} .
$$

We use classical notations for the set of real numbers, complex numbers, and integer numbers. The set of all nonnegative integer numbers will be denoted by $\mathbb{Z}_{+}$. 
It is known that if $\mathbf{T}=\{T(t)\}_{t \geq 0}$ is a strongly continuous semigroup on a complex Banach space $X$ and if $1 \leq p<\infty$ and $C_{p}>0$ are such that

$$
\int_{0}^{\infty}\|T(t) x\|^{p} d t \leq C_{p}\|x\|^{p}, \quad \forall x \in X
$$

then $\omega_{0}(\mathbf{T}) \leq-1 /\left(p C_{p}\right)$. See [2], where the case of semigroups acting on Hilbert spaces is analyzed, and see [3, pages 81-82] for the general case.

In this note, we extend this result to periodic strongly continuous evolution families acting on real or complex Banach spaces. Moreover, we prove the result with $p \in(0, \infty)$ instead of $1 \leq p<\infty$.

As an application of our theoretical results, we provide a simple example, which apparently cannot be treated using the results described above for semigroups.

A family $\mathcal{U}:=\{U(t, s)\}_{t \geq s \geq 0} \subset \mathscr{L}(X)$ is called a strongly continuous and $q$-periodic (for some $q \geq 1$ ) evolution family if it satisfies the following.

(1) $U(t, t)=I$ for all $t \geq 0$.

(2) $U(t, r) U(r, s)=U(t, s)$ for all $t \geq r \geq s \geq 0$.

(3) $U(t+q, s+q)=U(t, s)$ for all $t \geq s \geq 0$.

(4) The map $t \rightarrow U(t, s) x:[s, \infty) \rightarrow X$ is continuous for every $x \in X$ and is called exponentially bounded if there exist $\omega \in \mathbb{R}$ and $M_{\omega} \geq 1$, such that

$$
\|U(t, s)\| \leq M_{\omega} e^{\omega(t-s)} \quad \text { for } t \geq s \geq 0 .
$$

The growth bound $\omega_{0}(\mathcal{U})$ of an exponentially bounded evolution family $\mathcal{U}$ is the infimum of all $\omega \in \mathbb{R}$ for which there exists $M_{\omega} \geq 1$ such that (5) is fulfilled. It is known [4] that

$$
\omega_{0}(\mathcal{U})=\lim _{t \rightarrow \infty} \frac{\ln \|U(t, 0)\|}{t} .
$$

The family $\mathcal{U}$ is uniformly exponentially stable if its growth bound is negative. A $q$-periodic and strongly continuous evolution family $\mathscr{U}=\{U(t, s): t \geq s \geq 0\}$ is uniformly exponentially stable if and only if $\omega_{0}(\mathcal{U})$ is negative or, equivalently, if the spectral radius of the monodromy operator $U(q, 0)$ is less than one. The next result from the abstract theory of operators, originally given by Müller [5], will be useful in what follows.

Lemma 1. Let $X$ be a complex Banach space, and let $V \in$ $\mathscr{L}(X)$. If the spectral radius of $V$ is greater than or equal to 1 , then, for all $0<\varepsilon<1$ and any sequence $\left(a_{n}\right)$ with $a_{n} \rightarrow 0$ (as $n \rightarrow \infty)$ and $\left\|\left(a_{n}\right)\right\|_{\infty} \leq 1$, there exists a unit vector $u_{0} \in X$, such that

$$
\left\|V^{n} u_{0}\right\| \geq(1-\varepsilon) \cdot\left|a_{n}\right|, \quad \forall n \in \mathbb{Z}_{+} .
$$

Moreover, this result remains valid for real Banach spaces provided that the operator $V$ is power bounded; that is, $\sup \left\{\left\|V^{n}\right\|: n \in \mathbb{Z}_{+}\right\}$is finite. See [6] for further details.

\section{Preliminary Results}

The following two lemmas will be useful in the proof of Theorem 5 below. Its proof is essentially contained in [6, Theorem 1.2]. Because it has an interest in itself, we state it as a separate statement and infer its proof for the sake of completeness.

Lemma 2. Let $\xi:[0, \infty) \rightarrow[0,1]$ be a nonincreasing function such that $\lim _{t \rightarrow \infty} \xi(t)=0$. For each $\delta>0$, there exists a nonincreasing function $\rho:[0, \infty) \rightarrow[0,1]$, having the properties

(1) $\rho(t) \geq \xi(t)$, for all $t \geq 0$,

(2) $\lim _{t \rightarrow \infty} \rho(t)=0$,

(3) $\rho(t+s) \geq(1+\delta)^{-1} \rho(t) \rho(s)$, for all $t, s \geq 0$.

Proof. Let $0=K_{0}<K_{1}<K_{2} \ldots$ such that $0 \leq \xi(t) \leq(1+\delta)^{-n}$, when $t \geq K_{n}$. Next, choose the integers $0=R_{0}<R_{1}<R_{2} \ldots$ in such a way that $R_{n} \geq K_{n}$ for all $n$ and $R_{n}+R_{m} \leq R_{n+m}$ for all $m, n \geq 0$. For example, we can take $R_{n}=K_{0}+K_{1}+K_{2}+\cdots+K_{n}$.

Define the function $\rho:[0, \infty) \rightarrow[0,1]$ by

$$
\rho(t):=(1+\delta)^{-n} \quad \text { when } R_{n} \leq t<R_{n+1} .
$$

Clearly, $\rho$ is nonincreasing, $0 \leq \xi(t) \leq \rho(t) \leq 1$, and $\lim _{t \rightarrow \infty} \rho(t)=0$.

We claim that $\rho(t+s) \geq(1+\delta)^{-1} \rho(t) \rho(s)$ for all $t, s \geq 0$. Indeed, choose the integers $j_{t}$ and $j_{s}$ such that $R_{j_{t}} \leq t<R_{j_{t}+1}$, $R_{j_{s}} \leq s<R_{j_{s}+1}$. Then, $\rho(t)=(1+\delta)^{-j_{t}}$ and $\rho(s)=(1+\delta)^{-j_{s}}$. Now,

$$
t+s<R_{j_{t}+1}+R_{j_{s}+1} \leq R_{j_{t}+j_{s}+2}
$$

yields

$$
\rho(t+s) \geq(1+\delta)^{-j_{t}-j_{s}-1}=(1+\delta)^{-1} \rho(t) \rho(s) .
$$

Lemma 3. Let $\mathscr{U}=\{U(t, s)\}_{t \geq s \geq 0}$ be a strongly continuous and q-periodic evolution family on a complex Banach space $X$ such that $r(U(q, 0)) \geq 1$. Then, for any $0<\epsilon<1$ and every nonincreasing function $\xi:[0, \infty) \rightarrow[0,1]$, with $\lim _{t \rightarrow \infty} \xi(t)=0$, there exist $t_{0} \geq 0$ and a unit vector $x \in X$, such that

$$
\left\|U\left(t+t_{0}, t_{0}\right) x\right\| \geq(1-\epsilon) \xi(t) \quad \forall t \geq 0 .
$$

Proof.

Step 1. Define $\beta:[0, \infty) \rightarrow[0,1]$ by

$$
\beta(t):= \begin{cases}\xi(0), & \text { if } 0 \leq t<q, \\ \xi(t-q), & \text { if } t \geq q .\end{cases}
$$

The map $\beta(t)$ is nonincreasing, and $\lim _{t \rightarrow \infty} \beta(t)=0$.

Since $r(U(q, 0)) \geq 1$, there exists a norm one vector $x_{0} \in$ $X$, such that

$$
\left\|U^{k}(q, 0) x_{0}\right\| \geq \frac{1}{2} \beta(k) \quad \forall k \in \mathbb{Z}_{+} .
$$


This follows by Lemma 1 with $a_{n}=\beta(n), \epsilon=1 / 2$, and $V=$ $U(q, 0)$. For $t \geq 0$, let $N$ be an integer number, such that $N q \leq$ $t<N q+q$. We infer

$$
\begin{aligned}
\| U & (N q+q, 0) x_{0} \| \\
& =\left\|U(N q+q, t) U(t, 0) x_{0}\right\| \leq M\left\|U(t, 0) x_{0}\right\|,
\end{aligned}
$$

where $M=M_{\omega} e^{q \omega}$. Thus,

$$
\begin{aligned}
\left\|U(t, 0) x_{0}\right\| & \geq \frac{1}{M}\left\|U(N q+q, 0) x_{0}\right\| \\
& \geq \frac{1}{2 M} \beta((N+1)) \\
& \geq \frac{1}{2 M} \beta((N+1) q) \\
& =\frac{1}{2 M} \xi(N q) \\
& \geq \frac{1}{2 M} \xi(t) .
\end{aligned}
$$

Step 2. Let us choose $\delta>0$ such that $(1-\delta) /(1+\delta) \geq 1-\epsilon$. By Lemma 2, we can find a nonincreasing function $\rho:[0, \infty) \rightarrow$ $[0,1]$, such that $\rho(t) \geq \xi(t), \lim _{t \rightarrow \infty} \rho(t)=0$, and $\rho(t+s) \geq$ $(1+\delta)^{-1} \rho(t) \rho(s)$, for all $t, s \geq 0$.

In view of the first step, applied to the function $\rho$, we may choose a unit vector $y_{0} \in X$, such that

$$
\left\|U(t, 0) y_{0}\right\| \geq \frac{1}{2 M} \rho(t) \quad \forall t \geq 0 .
$$

Denote

$$
\eta:=\inf _{t \geq 0} \frac{\left\|U(t, 0) y_{0}\right\|}{\rho(t)}
$$

Then, $\eta \geq 1 /(2 M)$, and for all $t \geq 0$, we have that $\left\|U(t, 0) y_{0}\right\| \geq$ $\eta \rho(t)$. Thus,

$$
\sup _{t \geq 0} \frac{\eta \rho(t)}{\left\|U(t, 0) y_{0}\right\|}=1 \text {. }
$$

Now, let us choose $t_{0} \geq 0$, such that

$$
1-\delta \leq \frac{\eta \rho\left(t_{0}\right)}{\left\|U\left(t_{0}, 0\right) y_{0}\right\|}
$$

and let

$$
x:=\frac{U\left(t_{0}, 0\right) y_{0}}{\left\|U\left(t_{0}, 0\right) y_{0}\right\|} .
$$

We infer

$$
\begin{aligned}
\left\|U\left(t+t_{0}, t_{0}\right) x\right\| & =\frac{\left\|U\left(t+t_{0}, t_{0}\right) U\left(t_{0}, 0\right) y_{0}\right\|}{\left\|U\left(t_{0}, 0\right) y_{0}\right\|} \\
& =\frac{\left\|U\left(t+t_{0}, 0\right) y_{0}\right\|}{\left\|U\left(t_{0}, 0\right) y_{0}\right\|} \\
& \geq \frac{\eta \rho\left(t+t_{0}\right)}{\left\|U\left(t_{0}, 0\right) y_{0}\right\|} \\
& \geq \frac{\eta \rho(t) \rho\left(t_{0}\right)(1+\delta)^{-1}}{\left\|U\left(t_{0}, 0\right) y_{0}\right\|} \\
& \geq \frac{1-\delta}{1+\delta} \xi(t) \\
& \geq(1-\epsilon) \xi(t),
\end{aligned}
$$

which completes the proof.

Theorem 4. Let $\mathscr{U}=\{U(t, s)\}_{t \geq s \geq 0}$ be a strongly continuous and $q$-periodic evolution family on a complex Banach space $X$, and let $p \in(0, \infty)$. The following three statements are equivalent. (i) $\int_{X}^{\infty}\|U(t, 0) x\|^{p} d t \leq C_{p}<\infty$, for all unit vectors $x \in$

(ii) For every $s \geq 0$, one has

$$
\sup _{\|x\| \leq 1}\left(\int_{0}^{\infty}\|U(t+s, s) x\|^{p} d t\right):=C_{p}(s)<\infty .
$$

(iii) The growth bound, $\omega_{0}(\mathcal{U})$, is negative.

The equivalence of (i) and (iii) is given, for example, in [7, Theorem 3.3], and (ii) implying (i) is obvious. We have only to prove that (i) implies (ii). To do this, let $s=n q+r$, with $n \in \mathbb{Z}_{+}$and $r \in[0, q)$. Let $\omega<0$ and $M_{\omega} \geq 1$ such that

$$
\|U(t, s)\| \leq M_{\omega} e^{\omega(t-s)} \quad \forall t \geq s \geq 0 .
$$


Then,

$$
\begin{aligned}
& \int_{0}^{\infty}\|U(t+s, s) x\|^{p} d t \\
&=\int_{0}^{\infty}\|U(t+r, r) x\|^{p} d t \\
&=\int_{0}^{q-r}\|U(t+r, r) x\|^{p} d t \\
&+\int_{q-r}^{\infty}\|U(t+r, r) x\|^{p} d t \\
& \leq q M_{\omega}^{p} e^{p q \omega} \\
&+\int_{q-r}^{\infty}\|U(t+r-q, 0) U(q, r) x\|^{p} d t \\
&= q M_{\omega}^{p} e^{p q \omega}+\|U(q, r) x\|^{p} \\
& \times \int_{0}^{\infty}\left\|U(t, 0) \frac{U(q, r) x}{\|U(q, r) x\|}\right\|^{p} d t \\
& \leq q M_{\omega}^{p} e^{p q \omega}+C_{p} M_{\omega}^{p} e^{p q \omega} \\
& \leq M_{\omega}^{p} e^{p q \omega}\left(q+C_{p}\right) \\
&:= K_{p} \cdot
\end{aligned}
$$

\section{The Result and Its Consequences}

Theorem 5. Let $\mathcal{U}=\{U(t, s)\}_{t \geq s \geq 0}$ be a strongly continuous and $q$-periodic evolution family acting on a complex Banach space X. Suppose that the integral condition (22) is fulfilled for any $s \geq 0$ and some (and then for all) $0<p<\infty$. Then, there exists $t_{0} \geq 0$ such that $\omega_{0}(\mathscr{U}) \leq-1 /\left(p C_{p}\left(t_{0}\right)\right)$.

Proof. Let $\omega=\omega_{0}(\mathcal{U})$. The family

$$
\mathscr{B}:=\left\{B(t, s):=e^{-\omega(t-s)} U(t, s)\right\}_{t \geq s \geq 0}
$$

is a $q$-periodic evolution, family and $\omega_{0}(\mathscr{B})=0$. Let $\xi(t):=$ $e^{-\delta t}$ with $\delta>0$, and let $0<\epsilon<1$ be fixed. By applying Lemma 3 to the $q$-periodic evolution family $\mathscr{B}$, there exist $t_{0} \geq 0$ and a norm one vector $x \in X$, such that

$$
\left\|B\left(t+t_{0}, t_{0}\right) x\right\| \geq(1-\epsilon) e^{-\delta t}, \quad \forall t \geq 0,
$$

or equivalently

$$
\left\|U\left(t+t_{0}, t_{0}\right) x\right\| \geq(1-\epsilon) e^{(\omega-\delta) t}, \quad \forall t \geq 0 .
$$

Hence, via Theorem 4,

$$
\int_{0}^{\infty}(1-\epsilon)^{p} e^{(\omega-\delta) p t} d t \leq \int_{0}^{\infty}\left\|U\left(t+t_{0}, t_{0}\right) x\right\|^{p} d t \leq C_{p}\left(t_{0}\right) .
$$

That is, $-(1-\epsilon)^{p} /(\omega-\delta) p \leq C_{p}\left(t_{0}\right)$. Since $\epsilon>0$ and $\delta>0$ were arbitrary, the last inequality yields $-1 / p \omega \leq C_{p}\left(t_{0}\right)$ that is, $\omega_{0}(\mathcal{U}) \leq-1 /\left(p C_{p}\left(t_{0}\right)\right)$.
Corollary 6. Let $\mathscr{U}=\{U(t, s)\}_{t \geq s \geq 0}$ be a strongly continuous and $q$-periodic evolution family acting on a complex Banach space $X$. If $0<p<\infty$ and $C>0$ are such that

$$
\sup _{s \geq 0} \int_{0}^{\infty}\|U(t+s, s) x\|^{p} d t \leq C\|x\|^{p}, \quad \forall x \in X,
$$

then $\omega_{0}(\mathcal{U}) \leq-1 /(p C)$.

Corollary 7. Let $0<p<\infty$ and $\mathbf{T}=\{T(t)\}_{t \geq 0}$ be $a C_{0^{-}}$ semigroup on a Banach space $X$. If

$$
\sup _{\|x\| \leq 1} \int_{0}^{\infty}\|T(t) x\|^{p} d t=C_{p}<\infty
$$

then

$$
\omega_{0}(T) \leq-\frac{1}{p C_{p}} .
$$

Theorem 8. Let $\mathscr{U}=\{U(t, s)\}_{t \geq s \geq 0}$ be a strongly continuous and $q$-periodic evolution family acting on a real or complex Banach space $X$. Assume that $\omega_{0}:=\omega_{0}(\mathcal{U})$ is negative, and, in addition, the family

$$
\mathcal{U}_{0}:=\left\{e^{-\omega_{0}(t-s)} U(t, s): t \geq s \geq 0\right\}
$$

is uniformly bounded, and let $0<p<\infty$. Then, there exists $t_{0} \geq 0$ such that $\omega_{0}(\mathcal{U}) \leq-1 /\left(p C_{p}\left(t_{0}\right)\right)$, where $C_{p}\left(t_{0}\right)$ is defined in (22).

Actually, under the addition boundedness assumption, the monodromy operator associated with the family $\mathcal{U}_{0}$, that is, $e^{-q} U(q, 0)$, is power bounded, and we can use [6, Theorem 2.1].

\section{Some Examples and Remarks}

Now we present two examples which illustrate the theoretical result.

Example 1. Let $a: \mathbb{R} \rightarrow \mathbb{C}$ be a continuous and $q$-periodic function, and let us define $U(t, s):=e^{\int_{s}^{t} a(\tau) d \tau}$. Clearly, the family $\mathcal{U}:=\{U(t, s): t \geq s\}$ is a norm continuous $q$-periodic evolution family on $X:=\mathbb{C}$. If

$$
\sup _{s \in \mathbb{R}} \int_{0}^{\infty}\left(e^{\int_{s}^{s+t} \operatorname{Re}[a(\tau)] d \tau}\right)^{p} d t=C_{p}<\infty
$$

Then, for every $v>-1 /\left(p C_{p}\right)$ and every $t \geq s$, one has

$$
e^{\int_{s}^{t} \operatorname{Re}[a(\tau)] d \tau} \leq M_{\gamma} e^{\nu(t-s)} .
$$

Example 2. Let us choose $H:=L^{2}([0, \pi], \mathbb{C})$ to be the state space. Endowed with the usual inner product and norm, it becomes a complex Hilbert space, and, in addition, the one parameter family $\{T(t)\}_{t \geq 0}$, given by

$$
(T(t) x)(\xi)=\frac{2}{\pi} \sum_{n=1}^{\infty} e^{-t n^{2}} c_{n}(x) \sin n \xi, \quad \xi \in[0, \pi], t \geq 0,
$$


where $c_{n}(x):=\int_{0}^{\pi} x(s) \sin (n s) d s$, is a strongly continuous semigroup on $H$. The semigroup $\mathbf{T}=\{T(t)\}_{t \geq 0}$ is generated by the linear operator $A$ given by $A x=\ddot{x}$, and the maximal domain of $A$ is the set $D(A)$ of all $x \in H$ such that $x$ and $\dot{x}$ are absolutely continuous, $\ddot{x} \in H$, and $x(0)=x(\pi)=0$. Moreover, $T(t)$ is a self-adjoint operator for every $t \geq 0[8$, Example 1.3, pages 178, 198]. Consider the nonautonomous Cauchy problem

$$
\begin{gathered}
\frac{\partial z(t, \xi)}{\partial t}=f(t) \frac{\partial^{2} z(t, \xi)}{\partial^{2} \xi}, \quad t>0, \xi \in[0, \pi] \\
z(t, 0)=z(t, \pi)=0, \quad t \geq 0 \\
z(0, \xi)=x(\xi),
\end{gathered}
$$

where $x(\cdot)$ is a given function in $H$, and $f: \mathbb{R}_{+} \rightarrow[1, \infty)$ is a function having the following properties.

(1) $f(\cdot)$ is $q$-periodic for some $q \geq 1$.

(2) There exist $\alpha \in(0,1]$ and $c>0$ such that

$$
|f(t)-f(s)| \leq c|t-s|^{\alpha} \quad \forall t, s \geq 0 .
$$

Let $F(t)=\int_{0}^{t} f(t) d t$. As is well known, the solution $z(\cdot)$ of the above Cauchy problem satisfies the evolution condition

$$
z(t)=U(t, s) z(s)
$$

where $U(t, s)=T(F(t)-F(s))$. See [9, Example 2.9b].

We can estimate a positive constant $v$ having the property that the map $t \mapsto e^{v t}\|z(t)\|$ is bounded on $\mathbb{R}_{+}$.

Indeed,

$$
\begin{aligned}
& \int_{0}^{\infty}\|U(t, 0) x\|^{2} d t \\
& =\frac{2}{\pi} \int_{0}^{\infty} \sum_{n=1}^{\infty} c_{n}^{2}(x) e^{-2 n^{2} F(t)} d t \\
& =\frac{2}{\pi} \sum_{n=1}^{\infty} c_{n}^{2}(x) \int_{0}^{\infty} e^{-2 n^{2} F(t)} d t \\
& =\|x\|_{2}^{2} \int_{0}^{\infty} e^{-2 n^{2} F(t)} d t \\
& \leq\|x\|_{2}^{2} \int_{0}^{\infty} e^{-2 F(t)} d t .
\end{aligned}
$$

On the other hand

$$
\begin{aligned}
\int_{0}^{\infty} e^{-2 F(t)} d t & =\sum_{k=0}^{\infty} \int_{k q}^{(k+1) q} e^{-2 F(t)} d t \\
& =\sum_{k=0}^{\infty} \int_{0}^{q} e^{-2 F(k q+r)} d r \\
& =\sum_{k=0}^{\infty} e^{-2 k F(q)} \int_{0}^{q} e^{-2 F(r)} d r \\
& \leq q \sum_{k=0}^{\infty} e^{-2 k F(q)} \\
& =\frac{q e^{2 F(q)}}{e^{2 F(q)}-1} \\
& :=C_{2} .
\end{aligned}
$$

Hence,

$$
\int_{0}^{\infty}\|U(t, 0) x\|^{2} d t \leq C_{2}\|x\|_{2}^{2}
$$

Now by Theorem 8 , we get $\omega_{0}(\mathcal{U}) \leq-1 /\left(2 K_{2}\right)$, with $K_{2}$ being given in (24).

Remark 9. Even in the case of Hilbert spaces, the inequality

$$
\omega_{0}(\mathbf{T}) \leq-\frac{1}{p C_{p}}
$$

could be sharp.

Indeed, let $H:=l^{2}\left(\mathbb{Z}_{+}, \mathbb{C}\right)$ be the Hilbert space of all $\mathbb{C}$ valued sequences $x=\left(x_{n}\right)$ verifying

$$
\|x\|_{2}:=\left(\sum_{n=1}^{\infty}\left|x_{n}\right|^{2}\right)^{1 / 2}<\infty .
$$

Let $\varepsilon>0$ be fixed, and set

$$
(T(t) x)_{n}:=e^{-\varepsilon t} x_{n}, \quad n \in \mathbb{Z}_{+}, t \geq 0 .
$$

Obviously, the family $\mathbf{T}=\{T(t)\}_{t \geq 0}$ is a strongly continuous semigroup on $l^{2}\left(\mathbb{Z}_{+}, \mathbb{C}\right)$, and

$$
2 \omega_{0}(\mathbf{T}) \cdot\left(\sup _{\|x\|_{2} \leq 1} \int_{0}^{\infty}\|T(t) x\|_{2}^{2} d t\right)=-1 .
$$

Remark 10. It seems that the theoretical result of this paper cannot be applied to wave equations. We try to justify this statement in the following.

Let $A$ be a closed operator on a complex Banach space $X$, and let $x \in X$. Consider the Cauchy Problem

$$
P^{2}(x, 0)\left\{\begin{array}{l}
u^{\prime \prime}(t)=A u(t) \quad(t \geq 0) \\
u(0)=x \\
u^{\prime}(0)=0
\end{array}\right.
$$


Recall that a classical solution of $P^{2}(x, 0)$ is a function $u \in$ $C^{2}\left(\mathbb{R}_{+}, X\right)$ such that $u(t) \in D(A)$ for all $t \geq 0$, and $P^{2}(x, 0)$ holds. A function $u \in C\left(\mathbb{R}_{+}, X\right)$ is called a mild solution for $P^{2}(x, 0)$ if

$$
\begin{gathered}
\int_{0}^{t}(t-s) u(s) d s \in D(A), \\
u(t)=x+A \int_{0}^{t}(t-s) u(s) d s
\end{gathered}
$$

for all $t \geq 0$. Any $C^{2}\left(\mathbb{R}_{+}, X\right)$-valued mild solution of $P^{2}(x, 0)$ is a classical solution. The mild solution of $P^{2}(x, 0)$ leads to the notion of cosine function. See [10] or [11, pages 206-221], for further details.

Recall that a strongly continuous function cos : $\mathbb{R}_{+} \rightarrow$ $\mathscr{L}(X)$ is called cosine function if

(1) $\cos (0)=I$, where $I$ is the identity operator on $X$,

(2) $2 \cos (t) \cos (s)=\cos (t+s)+\cos (t-s)$ for all $t \geq 0$ and $s \geq 0$.

It is well known that any cosine function is exponentially bounded; that is, there are $\omega \in \mathbb{R}$ and $M>0$ such that $\|\cos (t)\| \leq M e^{\omega t}$ for all $t \geq 0$. Moreover, the uniform growth bound of any cosine function is nonnegative [11, Proposition 3.14.6]. Any cosine function has a generator. More exactly, there exist a positive $\omega$ and a linear operator $A$ such that $\left(\omega^{2}, \infty\right) \subset \rho(A)$ and

$$
\lambda R\left(\lambda^{2}, A\right)=\int_{0}^{\infty} e^{-\lambda t} \cos (t) d t \quad(\lambda>\omega) .
$$

See [11, Proposition 3.14.4]. On the other hand, if $A$ is a generator of a cosine function, the mild solution of the Cauchy Problem $P^{2}(x, 0)$ is given by $u(t)=\cos (t) x, t \geq 0$. Finally, we remind the reader that the result of this paper refers to exponentially stable evolution families, and then it cannot be applied to cosine functions.

\section{Acknowledgment}

The authors thank the anonymous referees for their useful suggestions on the preliminary version of this paper. In particular, the authors added the final remarks at the suggestion of the referees.

\section{References}

[1] R. Datko, "Uniform asymptotic stability of evolutionary processes in a Banach space," SIAM Journal on Mathematical Analysis, vol. 3, pp. 428-445, 1972.

[2] G. Weiss, "Weak $L^{p}$-stability of a linear semigroup on a Hilbert space implies exponential stability," Journal of Differential Equations, vol. 76, no. 2, pp. 269-285, 1988.

[3] J. van Neerven, The Asymptotic Behaviour of Semigroups of Linear Operators, vol. 88 of Operator Theory: Advances and Applications, Birkhäuser, Basel, Switzerland, 1996.

[4] C. Buşe and C. T. Drugan, “The numerical indexes associated to a q-periodic evolution families and integral characterizations for exponential stability," in Differential Equations and Applications, Y. J. Cho, J. K. Kim, and K. S. Ha, Eds., vol. 2, pp. 29-41, Nova Science, Huntington, New York, NY, USA, 2002.

[5] V. Müller, "Local spectral radius formula for operators in Banach spaces," Czechoslovak Mathematical Journal, vol. 38, no. 4, pp. 726-729, 1988.

[6] J. M. A. M. van Neerven, "On the orbits of an operator with spectral radius one," Czechoslovak Mathematical Journal, vol. 45, no. 3, pp. 495-502, 1995.

[7] C. Buşe and S. S. Dragomir, "A theorem of Rolewicz's type in solid function spaces," Glasgow Mathematical Journal, vol. 44, no. 1, pp. 125-135, 2002.

[8] J. Zabczyk, Mathematical Control Theory: An Introduction, Birkhäuser, Boston, Mass, USA, 1992.

[9] D. Daners and P. K. Medina, Abstract Evolution Equations, Periodic Problems and Applications, vol. 279 of Pitman Research Notes in Mathematics Series, Longman Scientific \& Technical, Harlow, UK, 1992.

[10] B. Nagy, "Cosine operator functions and the abstract Cauchy problem," Periodica Mathematica Hungarica, vol. 7, no. 3-4, pp. 213-217, 1976.

[11] W. Arendt, C. J. K. Batty, M. Hieber, and F. Neubrander, VectorValued Laplace Transforms and Cauchy Problems, vol. 96 of Monographs in Mathematics, Birkhäuser, Basel, Switzerland, 2001. 


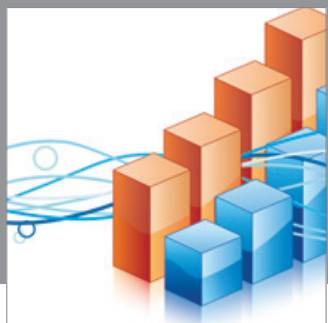

Advances in

Operations Research

mansans

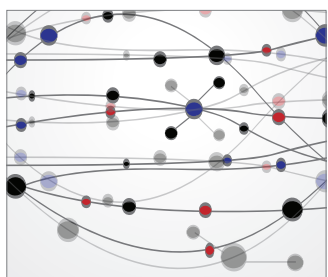

The Scientific World Journal
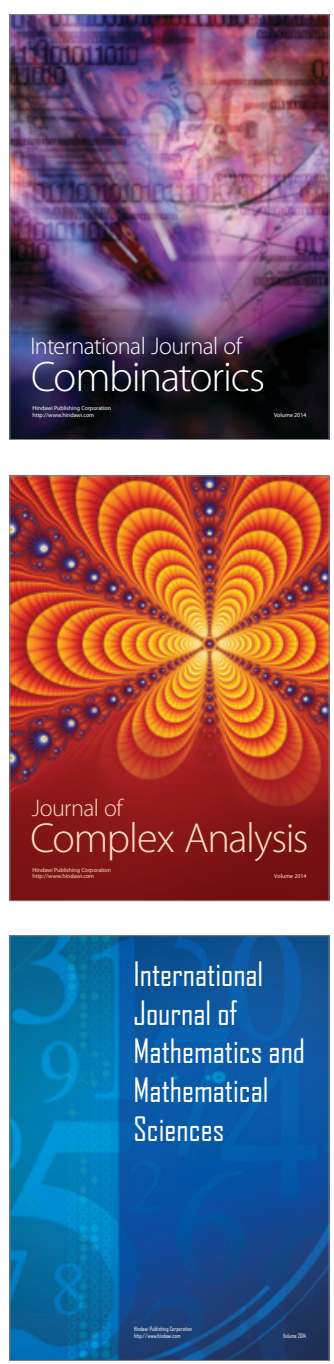
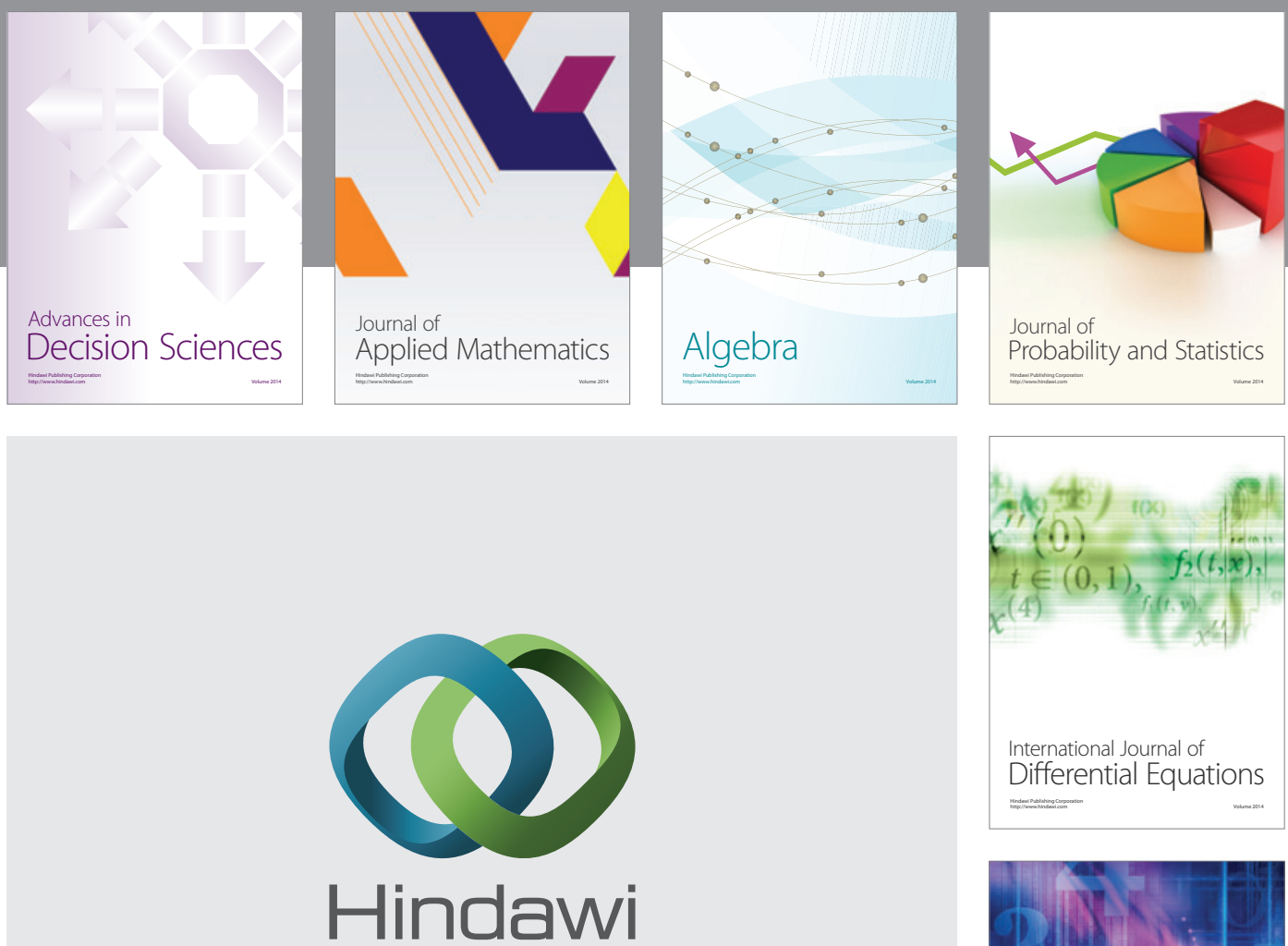

Submit your manuscripts at http://www.hindawi.com
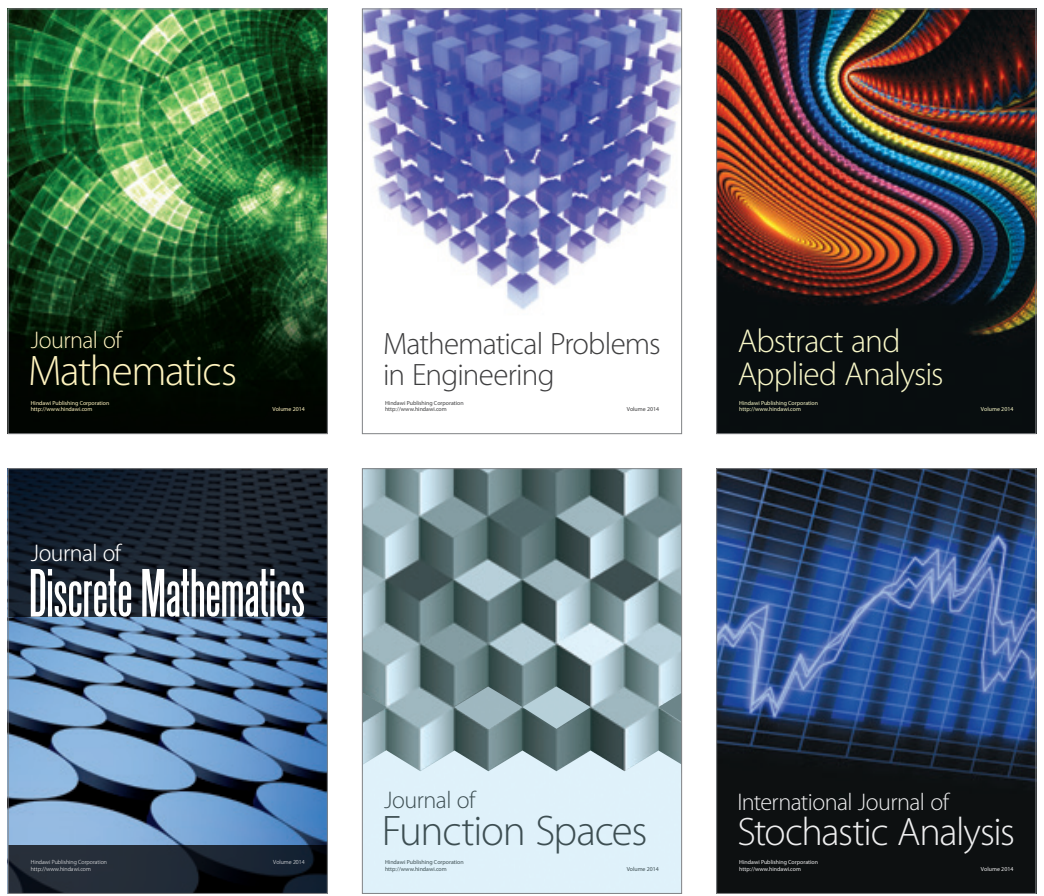

Journal of

Function Spaces

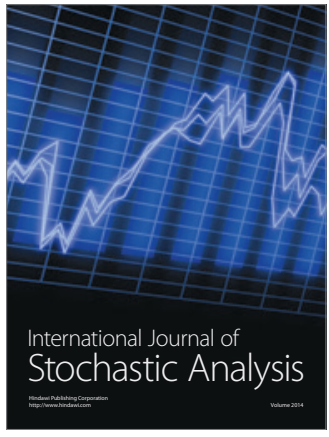

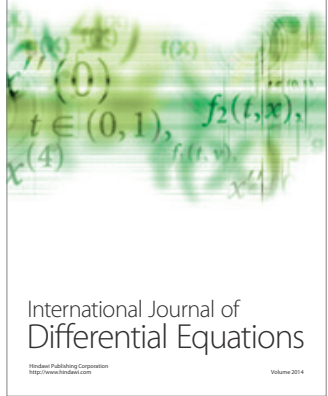
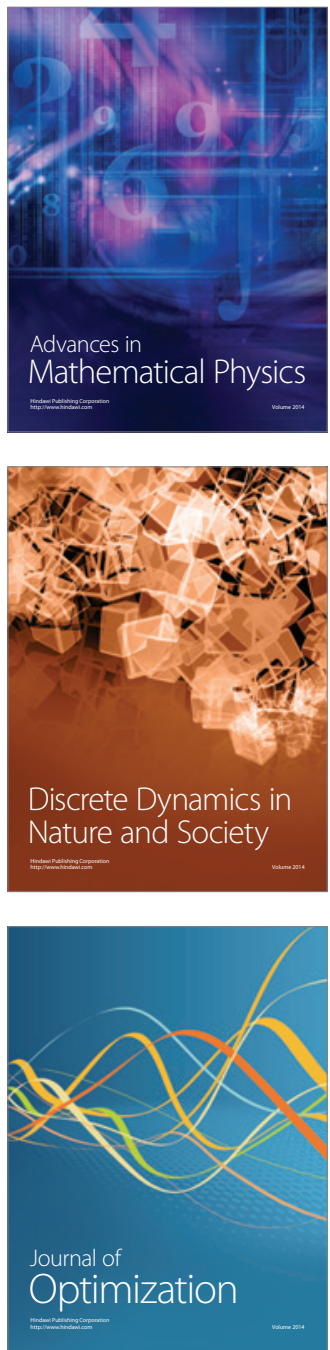